\section{Reliability of chest pain risk scores in cancer patients with suspected acute coronary syndrome}

\author{
Cansu Alyeşil', Serkan Yilmaz², İbrahim Ulaş Özturan³ ${ }^{3}$ Murat Pekdemir², \\ Elif Yaka², Nurettin Özgür Doğan²
}

'Department of Emergency Medicine, Mersin City Training and Research Hospital, Mersin, Turkey ${ }^{2}$ Department of Emergency Medicine, Faculty of Medicine, Kocaeli University, Kocaeli, Turkey ${ }^{3}$ Department of Emergency Medicine, Mersin Toros State Hospital, Mersin, Turkey

Objective The history, electrocardiogram, age, risk factors, troponin (HEART), the thrombolysis in myocardial infarction (TIMI), and Global Registry of Acute Coronary Events (GRACE) scores are useful risk stratification tools in the emergency department (ED). However, the accuracy of these scores in the cancer population is not well known. This study aimed to compare the performance of cardiac risk stratification scores in cancer patients with suspected acute coronary syndrome (ACS) in the ED.

Methods This prospective cohort study recruited patients with cancer who visited the ED because of suspected ACS. The development of any major adverse cardiac events (MACE) within 6 weeks was recorded, with the study outcome being a MACE within 6 weeks of ED admission.

Results A total of 178 patients participated in this study, of whom 5.6\% developed a MACE. Statistically significant differences were found between the mean HEART and TIMI scores in predicting MACE. The HEART score had the highest area under the curve $(0.64 ; 95 \%$ confidence interval, 0.48-0.81), highest sensitivity (80\%), and highest negative predictive value (97.5) in patients with cancer.

Conclusion We found a similar rate of MACE in cancer patients with low-risk chest pain compared to that in the general population. However, the HEART, TIMI, and GRACE scores had a lower performance in cancer patients with MACE compared to that in the general population.

Keywords Acute coronary syndrome; Neoplasms; Chest pain; Emergency service, hospital

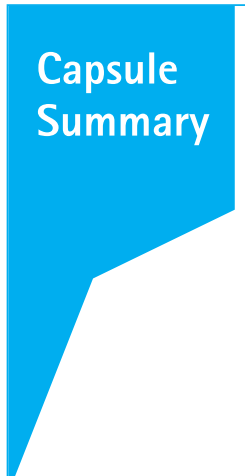

What is already known

The HEART (history, electrocardiogram, age, risk factors, troponin), TIMI (thrombolysis in myocardial infarction), and GRACE (Global Registry of Acute Coronary Events) scores are useful risk-stratification tools in the ED.

What is new in the current study

The accuracy of chest pain risk stratification scores in the cancer population is not well defined. The predictive value of chest pain risk stratification scores, including the HEART score, TIMI, and GRACE scores for MACE occurrence, in the cancer population was not good with an AUC value below 0.65 .
eISSN: 2383-4625

Received: 1 October 2019

Revised: 21 Decembeer 2019

Accepted: 26 December 2019

Correspondence to: İbrahim Ulas Özturan

Department of Emergency Medicine, Mersin Toros State Hospital, 33060, Mersin, Turkey

E-mail: i.ozturan@saglik.gov.tr ORCID

https://orcid.org/0000-0002-1364-5292

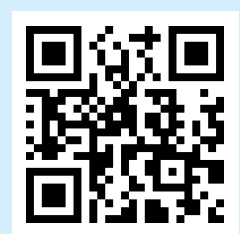

How to cite this article:

Alyeşil C, Yilmaz S, Özturan IU, Pekdemir M, Yaka E, Doğan NÖ. Reliability of chest pain risk scores in cancer patients with suspected acute coronary syndrome. Clin Exp Emerg Med 2020;7(4):275-280. https://doi. org/10.15441/ceem.19.088

This is an Open Access article distributed under the terms of the Creative Commons Attribution Non-Commercial License (https:// creativecommons.org/licenses/by-nc/4.0/). 


\section{INTRODUCTION}

Cancer and cardiovascular diseases are the two leading causes of morbidity and mortality worldwide. ${ }^{1}$ Nonspecific symptoms, such as shortness of breath, chest pain, palpitation, dizziness, and nausea, are commonly seen in patients with cancer. However, these symptoms can also be related to underlying acute coronary syndrome (ACS). ${ }^{2}$ Consequently, these nonspecific symptoms may mask the presence of ACS and lead to increased morbidity and mortality in patients with cancer.

According to the non-ST elevation ACS (NSTE-ACS) guidelines of the American Heart Association/American College of Cardiology NSTE-ACS, ${ }^{3}$ refractory angina, hemodynamic instability, and electrical instability in patients with NSTE-ACS are all indications of the need for coronary angiography for the reperfusion therapy. However, if these signs do not exist, additional strategies are necessary. Chest pain risk stratification scores, such as the history, electrocardiography (EKG), age, risk factors, troponin (HEART), the thrombolysis in myocardial infarction (TIMI), and Global Registry of Acute Coronary Events (GRACE) scores, as well as past medical history, EKG, troponin, age, and various risk factors are commonly used in the management of such patients. In addition to patient history and physical exam, these risk stratification tools help the clinicians to predict the risks and manage the patients accordingly.

Troponin I (Tnl) is a cardiac marker that is commonly used in risk stratification scores for chest pain. Although Tnl is a heartspecific enzyme, it may also be elevated in acute noncardiac critical illnesses. ${ }^{4}$ In the cancer population, additional comorbid diseases and cancer treatment itself can also lead to changes in Tnl levels. ${ }^{5}$ The clinical management of ACS can be influenced by changes in troponin levels, as well as by the expected survival of patients with cancer. In a previous study, it was reported that only a small number of cancer patients with ACS underwent revascularization treatments. Therefore, it might be a bias in recommending revascularization treatments in the cancer population considering the abnormal troponin level related to cancer and expected survival in the cancer population. ${ }^{6}$

Despite frequent admissions of cancer patients with symptoms suggestive of $\mathrm{ACS}_{1}{ }^{2}$ the emergency department (ED) management of this patient population is complicated by lack of consensus in the management of low risk ACS in the ED. Previous studies conducted on the general population with low risk chest pain reported a major adverse cardiac event (MACE) rate of $2 \% 0^{7,8}$ for a HEART score of $<3,1.7 \%$ for a TIMI score of $0,{ }^{9}$ and $2.2 \%$ for a GRACE score of $<87 .^{10}$ To our knowledge, no prior study has evaluated the validity and reliability of chest pain risk stratification scores in the cancer population. ${ }^{11}$ Therefore, this study aimed to evalu- ate the reliability of chest pain risk scores in cancer patients with suspected ACS in the ED.

\section{METHODS}

\section{Study design}

This prospective cohort study was conducted from September 2016 to June 2017 in the ED of an academic institution serving approximately 45,000 patients per year. Institutional review board approval and written informed consents from the patients were obtained prior to the commencement of the study (Kocaeli University, Board of Ethics on Non-invasive Clinical Human Studies Ethics Committee, KOÜ GOKEAK 2016/235).

\section{Study setting and population}

All patients admitted to the ED with a history of cancer and having symptoms suggesting ACS were evaluated for the study. Enrollment was performed consecutively, 7 days a week, and 24 hours a day. The inclusion criteria were as follows: age older than 18 years; having an active cancer; received chemotherapy or radiotherapy within the last 6 months; and presence of possible cardiac symptoms based on AHA case definitions, ${ }^{12}$ which include acute chest pain and/or epigastrium, neck pain, jaw pain, arm pain, or pressure without a significant noncardiac source. The exclusion criteria were as follows: ST-segment elevation myocardial infarction or left bundle branch block with a score of $>3$ according to the Sgarbossa Criteria; newly developed dysrhythmia; an impaired general condition requiring cardiopulmonary resuscitation; pregnancy; significant causes of noncardiac chest pain, such as known chest wall and rib metastasis; radiation therapy in the thoracic region; and/or unwillingness to participate in the study.

\section{Study protocol}

During the initial evaluation, a sample containing a minimum of $2 \mathrm{~mL}$ of blood was obtained to measure the $\mathrm{Tnl}$ levels; the sample was evaluated in the ED laboratory within 10 minutes of admission. Other required tests were performed at the discretion of the treating physician. The TIMI, HEART, and GRACE scores were calculated after the initial evaluation of the patients. For the TIMI score (0-7 points), the following were evaluated: age, coronary artery disease risk factors, known coronary stenosis, acetylsalicylate use in the past 7 days, presence of at least two episodes of severe angina in the last 24 hours, EKG ST changes, and cardiac biomarker levels. For the HEART score (0-10 points), the following were evaluated: the patient's history, EKG finding, age, risk factors, and initial troponin levels. For the GRACE score (2-383 points), the following were evaluated: age, heart rate, systolic 
blood pressure, creatinine level, cardiac arrest at admission, ST deviation on EKG, abnormal cardiac biomarkers, and Killip class.

MACE was defined as acute myocardial infarction, coronary revascularization, and cardiac death. Patients were followed up by phone 6 weeks after the first ED admission using a standardized questionnaire to determine whether a MACE occurred (01: Has the patient had a myocardial infarction in the last 6 weeks? 02: Has the patient had any percutaneous coronary intervention in the last 6 weeks? 03: Has the patient had any coronary artery bypass grafting surgery in the last 6 weeks? 04: How is the patient's current condition?). Hospital electronic medical records were also reviewed to confirm the occurrence of a MACE and, in cases that had died, the reason for death.

\section{Outcome measurement}

The study outcome was the presence of a MACE within 6 weeks of admission to the ED. The relationship between the MACE and cancer characteristics (primary cancer site, presence of metastasis, cancer stage, and cancer treatment within the last 6 months) was also described.

\section{Statistical analysis}

All statistical analyses were performed using IBM SPSS Statistics ver. 21.0 (IBM Corp., Armonk, NY, USA). Flauhalt's method is accepted as a valid test in studies investigating the value of diagnostic tests. ${ }^{13}$ Using this method, the expected sensitivity of HEART, $\mathrm{TIMI}$, and GRACE scores was 99\%, and the minimum lower confidence limit was taken as $95 \%$. The normality of the variables was determined using the Shapiro-Wilk test, histogram, and skewnesss

\section{Evaluated for the study}

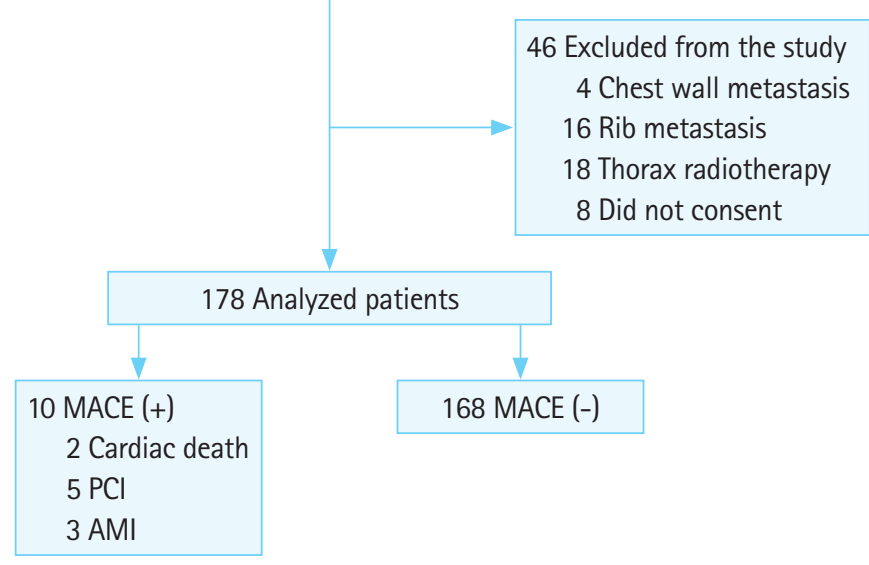

Fig. 1. Flow chart of patients with major adverse cardiac event (MACE) at the 6-week follow-up. $\mathrm{PCl}$, percutaneous coronary intervention; $\mathrm{AMI}$, acute myocardial infarction. and kurtosis analyses. The demographic and clinical characteristics of the patients were given as mean \pm standard deviation, median, interquartile range, 95\% confidence interval (Cl), and percentage (\%). The Student's t-test and Mann-Whitney U-test were used to compare the continuous variables, and the chi-squared test was used to compare the categorical variables. The diagnostic value of the HEART, TIMI, and GRACE scores for the determined threshold levels of each risk score was assessed using receiver operating characteristic analysis. Sensitivity, specificity, negative predictive values, and negative likelihood ratios were calculated and compared within 95\% $\mathrm{Cl}$ for these thresholds.

\section{RESULTS}

During the study period, 224 patients were evaluated for participation. After 46 patients were excluded, data from 178 patients were analyzed. A flow chart of the study participants is shown in Fig. 1.

Patient demographics are shown in Table 1. Patient mean age was $62.9(95 \% \mathrm{Cl}, 61.2$ to 64.7$)$ years. Of the 178 patients, 105 (59\%) were male. At the 6-week follow-up, 10 (5.6\%) patients had experienced a MACE. Of these cases, two resulted in cardiac death, five resulted in coronary revascularization, and three resulted in acute myocardial infarction. Both of the fatal cases were diagnosed with non-STE ACS. The reason for death was a resistant ventricular dysrhythmia. Of the patients with a MACE, 7 (70\%) had a history of smoking $(P=0.013)$.

The mean Tnl level in the MACE (+) and MACE (-) groups were $0.073 \pm 0.10$ and $0.022 \pm 0.057 \mathrm{ng} / \mathrm{mL}$, respectively. The mean dif-

Table 1. Patient demographics

\begin{tabular}{lllll}
\hline Characteristics & \multicolumn{1}{c}{$\begin{array}{c}\text { All patients } \\
(\mathrm{n}=178)\end{array}$} & $\begin{array}{c}\text { MACE }(+) \\
(\mathrm{n}=10)\end{array}$ & $\begin{array}{c}\text { MACE (-) } \\
(\mathrm{n}=168)\end{array}$ & $\begin{array}{c}\text { P- } \\
\text { value }\end{array}$ \\
\hline Sex, male & $105(59)$ & $7(70)$ & $98(58)$ & 0.529 \\
Age (yr) & $63(61.2-64.7)$ & $65(57.9-72.5)$ & $63(60.9-64.7)$ & 0.543 \\
History of CAD & $32(18)$ & $4(40)$ & $28(16.6)$ & 0.082 \\
Smoking & $57(32)$ & $7(70)$ & $50(23)$ & 0.013 \\
Family history of CAD & $15(8)$ & $2(20)$ & $13(8)$ & 0.201 \\
Hypertension & $81(45.5)$ & $5(50)$ & $76(45)$ & 1.000 \\
DM & $44(25)$ & $4(40)$ & $40(91)$ & 0.266 \\
Hypercholesterolemia & $42(23.6)$ & $3(30)$ & $39(24)$ & 0.702 \\
Obesity (BMI > 30) & $22(12)$ & $1(10)$ & $21(12.5)$ & 1.000 \\
Heart failure & $21(12)$ & $1(10)$ & $20(12)$ & 1.000 \\
Troponin (ng/mL) & $0.025 \pm 0.06$ & $0.07 \pm 0.10$ & $0.02 \pm 0.06$ & 0.011 \\
\hline
\end{tabular}

Values are presented as number (\%), median (interquartile range), or mean \pm standard deviation.

MACE, major adverse cardiac events; CAD, coronary artery disease; DM, diabetes mellitus; BMI, body mass index. 
Table 2. Average HEART, TIMI, and GRACE scores for patients with or without MACE

\begin{tabular}{lccccrr}
\hline & $\begin{array}{c}\text { All patients } \\
(\mathrm{n}=178)\end{array}$ & $\begin{array}{c}\text { MACE }(+) \\
(\mathrm{n}=10)\end{array}$ & $\begin{array}{c}\text { MACE }(-) \\
(\mathrm{n}=168)\end{array}$ & P-value & Mean difference & $95 \% \mathrm{Cl}$ \\
\hline HEART & $3.7 \pm 1.5$ & $4.6 \pm 1.8$ & $3.8 \pm 1.5$ & 0.016 & -1.22 & -2.2 to -0.2 \\
TIMI & $1.2 \pm 1.2$ & $2.1 \pm 1.8$ & $1.1 \pm 1.1$ & 0.002 & -1.20 & -1.9 to -0.4 \\
GRACE & $114.1 \pm 28.5$ & $116.2 \pm 21.8$ & $113.9 \pm 28.9$ & 0.800 & -2.48 & -21.8 to 16.8 \\
\hline
\end{tabular}

Values are presented as mean \pm standard deviation.

HEART, history, electrocardiogram, age, risk factors, troponin; TIMI, thrombolysis in myocardial infarction; GRACE, Global Registry of Acute Coronary Events; MACE, major adverse cardiac event; $\mathrm{Cl}$, confidence interval.

Table 3. Relationship between the cancer history and MACE in the study population

\begin{tabular}{|c|c|c|c|c|}
\hline & $\begin{array}{l}\text { All patients } \\
(n=178)\end{array}$ & $\begin{array}{l}\text { MACE }(+) \\
(n=10)\end{array}$ & $\begin{array}{l}\text { MACE }(-) \\
(n=168)\end{array}$ & $\begin{array}{c}\mathrm{P}- \\
\text { value }\end{array}$ \\
\hline \multicolumn{5}{|l|}{ Primary cancer site } \\
\hline Lung & $60(33.7)$ & $5(50.0)$ & $55(35.7)$ & 0.194 \\
\hline GIS & $40(22.5)$ & $3(30.0)$ & $37(22.0)$ & \\
\hline Brain & $3(1.7)$ & 0 & $3(1.8)$ & \\
\hline Breast & $12(6.7)$ & 0 & $12(7.1)$ & \\
\hline Kidney & $1(0.6)$ & 0 & $1(0.6)$ & \\
\hline Hematological & $18(10.1)$ & 0 & $18(10.7)$ & \\
\hline Gynecological & $17(9.6)$ & $1(10.0)$ & $16(9.5)$ & \\
\hline Prostate & $13(7.3)$ & $1(10.0)$ & $12(7.1)$ & \\
\hline Testis & $2(1.1)$ & 0 & $2(1.2)$ & \\
\hline Bladder & $5(2.8)$ & 0 & $5(3.0)$ & \\
\hline Other & $7(3.9)$ & 0 & $7(4.2)$ & \\
\hline Metastatic cancer & $118(56.2)$ & $7(70.0)$ & 111 (66.1) & 1.000 \\
\hline $\begin{array}{l}\text { Duration of diagnosis (mo), } \\
\text { median (IQR) }\end{array}$ & $10(4-30)$ & $5.5(2-14)$ & $12(3-36)$ & 0.185 \\
\hline \multicolumn{5}{|l|}{ Stage } \\
\hline 1 & $6(3.4)$ & $0(10.0)$ & $5(3.0)$ & 0.218 \\
\hline 2 & $27(16.1)$ & 0 & $27(16.1)$ & \\
\hline 3 & $16(9.0)$ & $3(20.0)$ & $14(8.3)$ & \\
\hline 4 & $129(72.5)$ & $7(70.0)$ & $122(72.6)$ & \\
\hline \multicolumn{5}{|l|}{ Treatment } \\
\hline Chemotherapy & 151 (84.8) & $7(70.0)$ & 144 (85.7) & 0.154 \\
\hline Radiotherapy & 94 (52.8) & $6(60.0)$ & $88(52.4)$ & 0.753 \\
\hline Surgery & $80(44.9)$ & $3(30.0)$ & 77 (45.8) & 0.348 \\
\hline Last treatment (day), median (IOR) & $10.5(1-30)$ & $29.5(10-78)$ & $10(0-25)$ & 0.022 \\
\hline
\end{tabular}

Values are presented as number (\%), median (interquartile range).

MACE, major adverse cardiac events; GIS, gastrointestinal system; IOR, interquartile range.

ference between the groups was $-0.05(95 \% \mathrm{Cl},-0.089$ to 0.011$)$.

The mean HEART, TIMI, and GRACE scores are summarized in Table 2. Statistically significant differences were found in the mean HEART and TIMI scores between the patients with and without a MACE $(P=0.016, P=0.02)$. However, there was no significant difference between the GRACE scores in patients with and without a MACE $(P=0.8)$.

Lung and gastrointestinal cancers were the most common cancer types among the study participants (34\% and 22\%, respec-

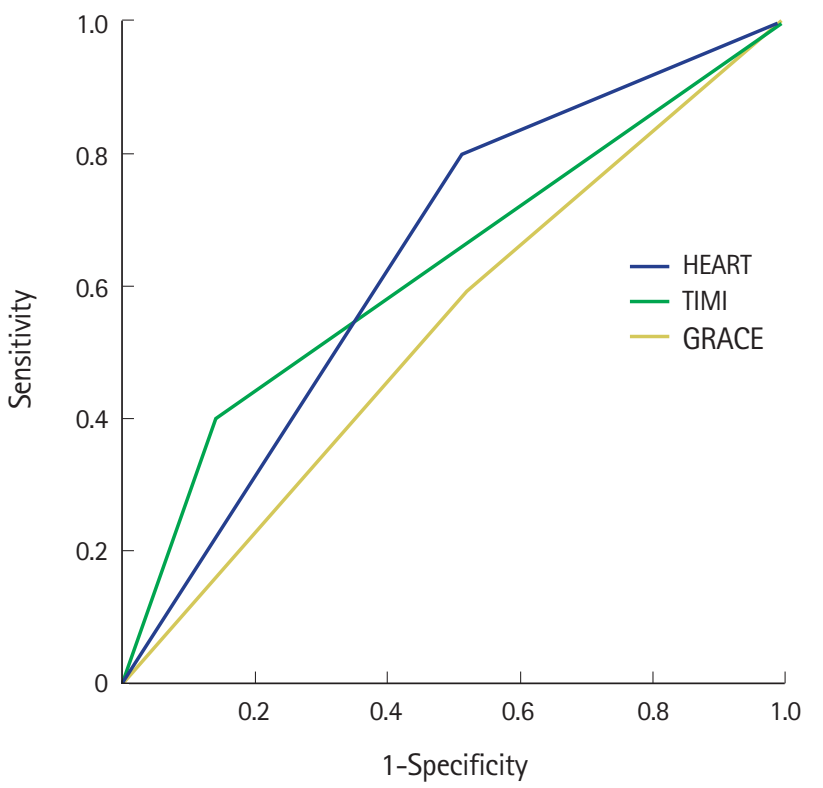

Fig. 2. Receiver operating characteristic curve showing the prediction of major adverse cardiac event by the history, electrocardiogram, age, risk factors, troponin (HEART), thrombolysis in myocardial infarction (TIMI), and Global Registry of Acute Coronary Events (GRACE) scores within 6 weeks.

tively). No statistically significant difference was found in the cancer type, cancer stage, treatment of cancer between the patients with and without a MACE $(P>0.05)$ (Table 3).

The receiver operating characteristic curve showing the MACE prediction according to the participants' HEART, TIMI, and GRACE scores 6 weeks after ED admission is shown in Fig. 2. The area under curve values for HEART, TIMI, and GRACE scores were 0.64 (95\% Cl, 0.48 to 0.81 ), 0.63 ( $95 \% \mathrm{Cl}, 0.43$ to 0.83 ), and 0.54 (95\% $\mathrm{Cl}, 0.36$ to 0.72$)$, respectively. A comparison of the validity and reliability of the HEART, TIMI, and GRACE scores for low-risk patients is presented in Table 4. MACE occurred in 2 of 84 patients $(2.4 \%)$ with a HEART score of $\leq 3$, in 6 of 150 patients (4\%) with a TIMI score of $\leq 2$, and in 3 of 84 patients (3.6\%) with a GRACE score of $<110$. When the validity and reliability of the HEART, TIMI, and GRACE scores were compared at the 6-week follow-up, it was found that the HEART score had the highest sensitivity and 
Table 4. Test performance of HEART, TIMI, and GRACE scores in a cancer population

\begin{tabular}{lcccccc}
\hline & $\begin{array}{c}\text { Sensitiv- } \\
\text { ity } \%\end{array}$ & $\begin{array}{c}\text { Specific- } \\
\text { ity } \%\end{array}$ & PPV $\%$ & NPV \% & LR+ & LR- \\
\hline HEART $\leq 3$ & 80 & 48.1 & 9.3 & 97.5 & 1.54 & 0.42 \\
TIMI $\leq 2$ & 40 & 86 & 14.3 & 96 & 2.85 & 0.70 \\
GRACE $\leq 110$ & 60 & 47.6 & 6.3 & 95.2 & 1.14 & 0.84
\end{tabular}

HEART, history, electrocardiogram, age, risk factors, troponin; TIMI, thrombolysis in myocardial infarction; GRACE, Global Registry of Acute Coronary Events; PPV, positive predictive value; NPV, negative predictive value; $L R+$, positive likelihood ratio; LR-, negative likelihood ratio.

the highest negative predictive value in cancer patients with symptoms suggestive of an ACS in the ED (Table 4).

\section{DISCUSSION}

This study compared the ability of HEART, TIMI, and GRACE scores to predict the occurrence of a MACE in patients with cancer at 6 weeks after an ED admission for low-risk chest pain. The HEART score was found to have the highest sensitivity and the highest negative predictive value in this patient population. However, all three scores were found to have lower performance in patients with cancer than in the general population.

Several studies have compared chest pain risk stratification scores. Sakamoto et al. ${ }^{8}$ compared the HEART, TIMI, and GRACE scores in patients with acute chest pain in the ED; they found that the HEART score had the highest performance in terms of 30-day MACE prediction (AUC of the HEART, TIMI, and GRACE scores were $0.78,0.65$, and 0.62 , respectively). Poldervaart et al. ${ }^{10}$ also compared HEART, TIMI, and GRACE scores and reported higher performance of the HEART score compared to the other two scores (AUC: $0.86,0.80$, and 0.73 , respectively). However, the ability of these scores to predict MACE in the cancer population remained unclear. The present study found a lower sensitivity and negative predictive value of the HEART, TIMI, and GRACE scoring systems for MACE in cancer patients compared to the general population. This lower performance could be related to various confounding and vague symptoms experienced by patients with cancer, as well as abnormal troponin or creatinine levels associated with the cancer or the treatment.

Previous studies identified the factors having effects on the pathogenesis of ACS development in cancer patients such as radiotherapy, chemotherapy, cancer site, presence of metastasis or cancer stage. ${ }^{14-19}$ However, there was no significant relationship between these factors and ACS development in cancer patients in this study. Yet, it is important to note that all of the patients with a MACE had advanced-stage (stage 3-4) cancer. Clinical problems in patients with advanced-stage cancer, such as anemia, hypotension, increased treatment burden, and comorbidities, might explain the development of MACE.

To the best of our knowledge, there is no clear data showing that cancer directly influences the troponin levels in patients with cancer. Increases in troponins after chemotherapy have been observed in children receiving anthracycline and in patients with hematological malignancies. ${ }^{20,21}$ In our study, none of the patients were treated with anthracycline therapy. Cardinale et al. ${ }^{22}$ showed $\mathrm{Tnl}$ to be a sensitive and specific marker of myocardial damage after high-dose chemotherapy, as well as a predictor of the onset and severity of ventricular dysfunction. It has also been reported that, in the majority of patients with high levels of Tnl, a significant reduction in left ventricular ejection fraction occurs within the first year of high-dose chemotherapy. Although we did not categorize the patients in this study according to chemotherapy agents and could not show a significant relationship between chemotherapy and MACE, the mean Tnl level was significantly higher in the MACE group.

This study has several limitations. First, the final evaluation of ACS and the decision of definitive treatments, such as percutaneous transluminal coronary angioplasty, were determined by different cardiologists. This may lead to personal differences in the management of cancer patients with ACS. Predicted survival of these patients may influence decisions for patient care. Second, radiation-induced coronary artery disease is a common cause of mortality in patients with cancer who undergo radiation therapy in the thoracic region. ${ }^{16}$ Because patients who had radiation therapy in the thoracic region were excluded in this study, we could not analyze the data from patients with radiation-induced coronary artery disease. Finally, our study population was smaller than that of similar studies that used Flauhalt's method to calculate sample size. Although the rate of MACE in low risk patient in our study was similar to that reported in the literature, the small number of detected MACE cases may prevent generalization of the results.

In summary, this study found a similar rate of MACE occurring in patients with cancer and low-risk chest pain compared to the general population. However, the HEART, TIMI, and GRACE scores were found to have lower predictive ability for MACE in patients with cancer. More reliable risk assessment tools should be applied to provide optimal care for cancer patients with suspected ACS in the ED.

\section{CONFLICT OF INTEREST}

No potential conflict of interest relevant to this article was reported. 


\section{REFERENCES}

1. Fuster V, Voute J. MDGs: chronic diseases are not on the agenda. Lancet 2005;366:1512-4.

2. Rivera DR, Gallicchio L, Brown J, Liu B, Kyriacou DN, Shelburne $\mathrm{N}$. Trends in adult cancer-related emergency department utilization: an analysis of data from the nationwide emergency department sample. JAMA Oncol 2017;3:e172450.

3. Amsterdam EA, Wenger NK, Brindis RG, et al. 2014 AHA/ACC guideline for the management of patients with non-ST-elevation acute coronary syndromes: a report of the American College of Cardiology/American Heart Association Task Force on Practice Guidelines. Circulation 2014;130:e344-426.

4. Lin YN, Chang YJ, Chen YH, et al. Nationwide population-based cohort study on the association of acute coronary syndrome in patients with malignancies. Support Care Cancer 2014;22: 2707-13.

5. Cardinale D, Sandri MT, Colombo A, et al. Prognostic value of troponin I in cardiac risk stratification of cancer patients undergoing high-dose chemotherapy. Circulation 2004;109:274954.

6. Yusuf SW, Daraban N, Abbasi N, Lei X, Durand JB, Daher IN. Treatment and outcomes of acute coronary syndrome in the cancer population. Clin Cardiol 2012;35:443-50.

7. Backus BE, Six AJ, Kelder JC, et al. A prospective validation of the HEART score for chest pain patients at the emergency department. Int J Cardiol 2013;168:2153-8.

8. Sakamoto JT, Liu N, Koh ZX, et al. Comparing HEART, TIMI, and GRACE scores for prediction of 30-day major adverse cardiac events in high acuity chest pain patients in the emergency department. Int J Cardiol 2016;221:759-64.

9. Chase M, Robey JL, Zogby KE, Sease KL, Shofer FS, Hollander JE. Prospective validation of the Thrombolysis in Myocardial Infarction Risk Score in the emergency department chest pain population. Ann Emerg Med 2006;48:252-9.

10. Poldervaart JM, Langedijk M, Backus BE, et al. Comparison of the GRACE, HEART and TIMI score to predict major adverse cardiac events in chest pain patients at the emergency department. Int J Cardiol 2017;227:656-61.

11. Carlton EW, Khattab A, Greaves K. Identifying patients suitable for discharge after a single-presentation high-sensitivity troponin result: a comparison of five established risk scores and two high-sensitivity assays. Ann Emerg Med 2015;66: 635-45.
12. Luepker RV, Apple FS, Christenson RH, et al. Case definitions for acute coronary heart disease in epidemiology and clinical research studies: a statement from the AHA Council on Epidemiology and Prevention; AHA Statistics Committee; World Heart Federation Council on Epidemiology and Prevention; the European Society of Cardiology Working Group on Epidemiology and Prevention; Centers for Disease Control and Prevention; and the National Heart, Lung, and Blood Institute. Circulation 2003;108:2543-9.

13. Flahault A, Cadilhac M, Thomas $G$. Sample size calculation should be performed for design accuracy in diagnostic test studies. J Clin Epidemiol 2005;58:859-62.

14. Jones $D$, Wismayer $K$, Bozas $G$, Palmer J, Elliott M, Maraveyas A. The risk of venous thromboembolism associated with peripherally inserted central catheters in ambulant cancer patients. Thromb J 2017;15:25.

15. Blann AD, Dunmore S. Arterial and venous thrombosis in cancer patients. Cardiol Res Pract 2011;2011:394740.

16. Cuomo JR, Sharma GK, Conger PD, Weintraub NL. Novel concepts in radiation-induced cardiovascular disease. World J Cardiol 2016;8:504-19.

17. Lipshultz SE, Adams MJ, Colan SD, et al. Long-term cardiovascular toxicity in children, adolescents, and young adults who receive cancer therapy: pathophysiology, course, monitoring, management, prevention, and research directions: a scientific statement from the American Heart Association. Circulation 2013;128:1927-95.

18. Kupeli S, Hazirolan T, Varan A, et al. Evaluation of coronary artery disease by computed tomography angiography in patients treated for childhood Hodgkin's lymphoma. J Clin Oncol 2010;28:1025-30.

19. Min SS, Wierzbicki AS. Radiotherapy, chemotherapy and atherosclerosis. Curr Opin Cardiol 2017;32:441-7.

20. Lipshultz SE, Rifai N, Sallan SE, et al. Predictive value of cardiac troponin $\mathrm{T}$ in pediatric patients at risk for myocardial injury. Circulation 1997;96:2641-8.

21. Missov E, Calzolari C, Davy JM, Leclercq F, Rossi M, Pau B. Cardiac troponin I in patients with hematologic malignancies. Coron Artery Dis 1997;8:537-41.

22. Cardinale D, Sandri MT, Martinoni A, et al. Left ventricular dysfunction predicted by early troponin I release after high-dose chemotherapy. J Am Coll Cardiol 2000;36:517-22. 